\title{
FPGA implementation of Wavelet Neural Network for epileptic seizure detection
}

\author{
Hari Kumar $\mathbf{R}^{1 \mathrm{a})}$, Balasubramani $\mathbf{M}^{2}$, and Ganesh Babu ${ }^{1}$ \\ ${ }^{1}$ Professor, ECE Department, Bannari Amman Institute of Technology, \\ Sathyamangalam, India \\ ${ }^{2}$ Research Scholar, ECE Department, Bannari Amman Institute of Technology, \\ Sathyamangalam, India \\ a) rishiveda@yahoo.com
}

Abstract: This paper introduces an implementation of Wavelet Neural Network (WNN) with Particle Swarm Optimization (PSO) learning ability on Field Programmable Gate Array for detection of severity index of epileptic seizure detection. The Electroencephalography (EEG) signals were first pre-processed using discrete wavelet transforms (DWTs). This was followed by the feature selection stage, where five wavelet parameters and four representative summary statistics were computed. Four different activation functions were used in the hidden nodes of WNNs. The best combination to be used was the WNNs that employed Haar wavelet as the activation function, with Haar wavelet along with Heursure soft thresholding at the feature extraction stage. The PSO learning method is used in this paper. Twenty known epilepsy patients in nine defined groups are studied. The efficacy of the WNN is analyzed through sensitivity, specificity and classification accuracy. Higher the benchmark values will be the better classifier.

Keywords: FPGA, Wavelet Neural Networks (WNN), Particle Swarm Optimization (PSO), epilepsy, wavelet transform

Classification: Integrated circuits

\section{References}

[1] H. Adeli, Z. Zhou and N. Dadmehr: Journal of Neuroscience Methods 123 (2003) 69.

[2] A. Subasi: Expert Systems with Applications 28 (2005) 701.

[3] G. J. Gibson, S. Siu and C. F. N. Cowen: Proc. IEEE Conf. Acoust. Speech Signal Process. (1989) 1183.

[4] K. Hornik: Neural Networks 2 (1989) 359.

[5] Z. L. Gaing: IEEE Trans. Energy Convers. 19 (2004) 384.

[6] R. Mendes, P. Cortez, M. Rocha and J. Neves: The 2002 International Joint Conference on Neural Networks (2002) 1895.

[7] N. M. Botros and M. Abdul-Aziz: IEEE Trans. Ind. Electron. 41 (1994) 665 . 


\section{Introduction}

Approximately $1 \%$ of the world population suffers from epilepsy [1]. The recognition of epileptiform discharges occurring in the EEG between seizures is a principal component in the diagnosis of epilepsy $[1,2]$. Artificial neural networks (ANNs) have been extensively used in many biomedical signal analysis. The most commonly employed ANN is the multilayer perceptron (MLP) proposed by Gibson et al [3]. The multilayer perceptron (MLP) provided with general transfer functions active over a large scale of input values has been widely employed to achieve global approximations [4]. In this study, a novel hybrid system was proposed. The abrupt changes in the EEG signals can be traced and studied using DWT. WNNs were adopted as the statistical models because of their faster learning rate. WNN trains their parameters iteratively employing a learning algorithm. PSO algorithm is introduced to attain global optimum ability. Each particle in a swarm shares the information among themselves. The PSO toil as a classifier with high predictive accuracy for epileptic seizure detection. This paper addresses the FPGA Synthesis of WNN model towards classification of severity index of epileptic seizure detection.

\section{Feature extraction}

Four types of wavelets, namely Haar, Sym8, Daubechies wavelet of order 2 (dB2) and Daubechies wavelet order $4(\mathrm{~dB} 4)$ were considered for feature extraction at pre-processing stage. Through four-level wavelet decomposition, the signals were decomposed into coarse approximation (a) and detail information (d). After extraction of wavelet coefficient, a total of four statistics were derived from the produced wavelet coefficients. They are minimum, maximum and mean of the absolute values of the generated wavelet coefficients in each sub-band and standard deviations of the generated wavelet coefficients from each sub-band [7]. Totally nine features are extracted which includes five wavelet coefficients and four statistical parameters. In order to ensure the good feature extraction and removal of the noisy parameters, we used hard thresholding method and four types of soft thresholding such as Heursure, Minimaxi, Rigrsure and Sqtwolog. Hence the obtained features are cleaned without any noisy components. They represent better extracted feature of the samples. The twenty patients are labelled into nine groups based upon their EEG signal and clinical observation. The same is shown in the Table I. The patients are

Table I. Grouping of Twenty Patients Based on EEG Signals and Clinical Observation.

\begin{tabular}{clc}
\hline Group & Patient No & Target Value \\
\hline G1 & $1,2,11,20$ & 0.85 \\
\hline G2 & $3,7,14,18$ & 0.65 \\
\hline G3 & 4,13 & 0.45 \\
\hline G4 & 6,12 & 0.35 \\
\hline G5 & $5,8,19$ & 0.25 \\
\hline G6 & 15,17 & 0.20 \\
\hline G7 & 16 & 0.15 \\
\hline G8 & 10 & 0.10 \\
\hline G9 & 9 & 0.05
\end{tabular}


assigned and labelled in groups based on the severity index or Target values of the frequent occurrence of epileptic seizures. The patients who are labeled in the G1 group are considered to be more vulnerable one and G9 group as least disturbed one.

Hardware implementation of Wavelet Neural Network is discussed in the following section the paper

\section{Hardware implementation of Wavelet Neural Network}

The WNN consist of three layers-the input layer: receives the input data, the hidden layer: performs the nonlinear mapping and the output layer: determines the class of the input data. The equation that describes the modeling is given by the following equation:

$$
y(X)=\sum_{i=1}^{n} w i j \frac{1}{\sqrt{|d|}}\left(\frac{X-t i}{d}\right)
$$

Where $\frac{1}{\sqrt{|d|}}\left(\frac{X-t i}{d}\right)$ is the wavelet function, $\mathrm{x}$ is the input vector, $\mathrm{n}$ is the number of hidden nodes, $y$ is the output, $j$ is the number of output nodes, $\mathrm{w}$ is the weight matrix that minimizes the error goal, $\mathrm{d}$ is the dilation parameters vector and $\mathrm{t}$ is the translation parameters vector. Haar, Sym8, $\mathrm{dB} 2$ and $\mathrm{dB} 4$ wavelets are once again used as activation function. Since the wavelet function is a nonlinear activation function, it is difficult to implement using the hardware directly. In this paper, we use the Taylor series and LUT to approximate the wavelet function. Fig. 1. shows The Overall Component of the Wavelet Layer

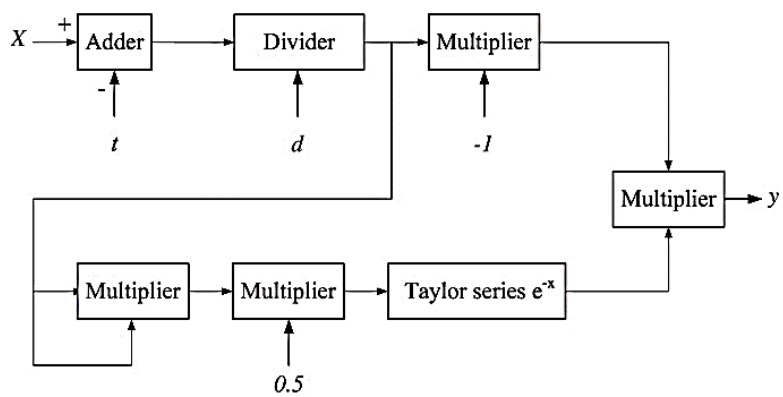

Fig. 1. The Overall Component of the Wavelet Layer.

\subsection{Particle Swarm Optimization (PSO)}

Particle Swarm Optimization (PSO) is a good optimizer that holds several highly eligible attributes, including the case that the basic algorithm is easy to understand and to implement [5]. A collection of particles are defined, where each particle is determined a random position in the $\mathrm{N}$-dimensional problem space. Each particle's position has a close similarity to a candidate solution to the optimization problem. Each of these particle positions is scored to gain a fitness value based on how well it solves the problem. Applying the local best position - Lbest and the global best position Gbest, a new velocity for all the particles is updated in equation 2 . 


$$
\begin{aligned}
& \overleftarrow{v_{i}}(k+1)-\omega * \overleftarrow{v_{i}}(k)+\varphi_{1} * \operatorname{rand}() *\left(\text { Lbest }-\overleftarrow{x_{i}}(k)\right)+\varphi_{2} \\
& \quad * \operatorname{rand}() *\left(\text { Gbest }-\overleftarrow{x_{i}}(k)\right)
\end{aligned}
$$

Where $\omega, \varphi_{1}, \varphi_{2}$ are called the coefficient of inertia, cognitive and society. The rand () is uniformly distributed random numbers in $[0,1]$. The term $\overrightarrow{v_{i}}$ is limited to the range $\pm \overrightarrow{v_{\max }}$. If the velocity infringes this limit, it will be set to its proper limit. Varying velocity empowers every particle to search around its individual local best position - Lbest and the global best position - Gbest. Particle changes its position depending on the updated velocities: When every particle is updated, the fitness value of each particle is evaluated again [6]. If the fitness value of the new particle is greater than those of local best, then the local best will be changed with the new particle. If the fitness value of the new particle is greater than those of global best, then the global best will be also changed with the new particle [6]. The PSO algorithm repeats the updating process step by step, the entire population evolves toward the optimum result. Fig. 2. shows the Block Diagram for the Hardware Implementation of PSO Algorithm.

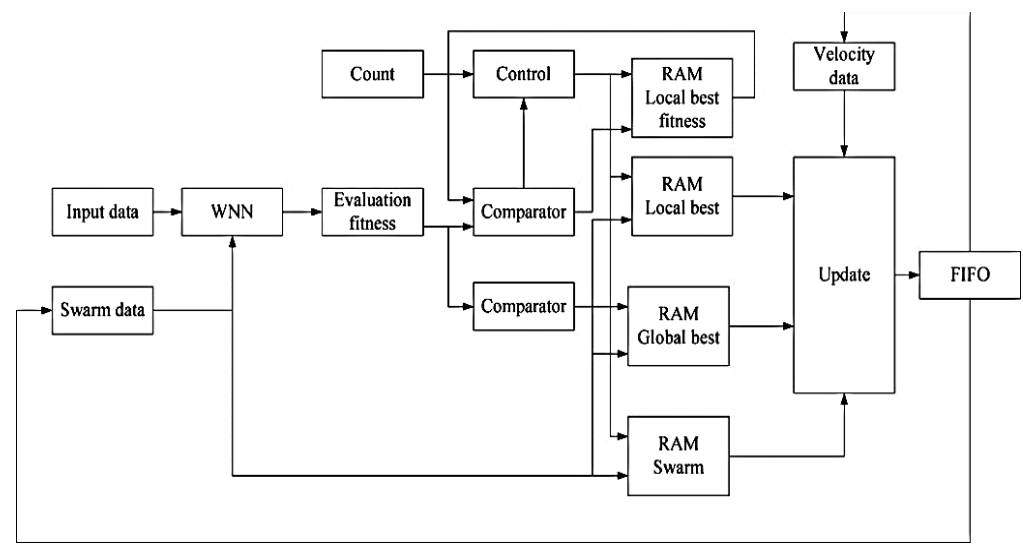

Fig. 2. The Block Diagram for the Hardware Implementation of PSO Algorithm.

\section{Results and discussion}

A group of twenty patients are examined in this study. The results are analyzed through Mean Square Error Value (MSE) which is defined as [5]

$$
M S E=\frac{1}{N} \sum_{i=1}^{n}\left(T_{j}-Q_{j}\right)^{2}
$$

Where, $T_{j}=$ Target and value and $Q_{j}=$ Obtained output value. The performance of WNN is analyzed through the bench mark parameters such as sensitivity, specificity and average detection. Table II. shows the performance analysis of twenty patients in Hard Thresholding method based on the benchmark parameters. In terms of average detection Haar Wavelet is ranked at high level than other three wavelets. Even though Sym8 wavelet has low MSE value but settled at low value of average detection due to its inherent problem of less specificity.

Table III. shows the performance analysis of twenty patients against four wavelets with four Soft Thresholding methods. Here also Haar wavelet 
Table II. Performance Analysis of Twenty Patients in Hard Thresholding.

\begin{tabular}{lllll}
\hline Wavelets & Sensitivity & Specificity & $\begin{array}{l}\text { Average } \\
\text { Detection }\end{array}$ & MSE \\
\hline Haar & 92.64795 & 96.66195 & 94.655 & 0.007038 \\
\hline $\mathrm{dB} 2$ & 92.664 & 95.64911 & 94.15679 & 0.007086 \\
\hline $\mathrm{dB} 4$ & 93.11347 & 95.26684 & 94.19005 & 0.008232 \\
\hline Sym8 & 93.73974 & 93.86884 & 93.80437 & 0.005421 \\
\hline
\end{tabular}

Table III. Performance Analysis of Twenty Patients in Soft Thresholding.

\begin{tabular}{clllll}
\hline \multirow{2}{*}{ Wavelets } & \multirow{2}{*}{ Parameters } & \multicolumn{5}{l}{ Soft Thresholding } \\
\cline { 3 - 6 } & & Heursure & Minimaxi & Rigrsure & Sqtwolog \\
\hline \multirow{3}{*}{ Haar } & Sensitivity & 94.7454 & 92.3624 & 93.1344 & 91.0101 \\
\cline { 2 - 6 } & Specificity & 97.9304 & 95.8607 & 96.3612 & 93.0823 \\
\cline { 2 - 6 } & Average Detection & 96.3380 & 94.1116 & 94.7478 & 92.0462 \\
\cline { 2 - 6 } & MSE & 0.00584 & 0.00594 & 0.00348 & 0.00403 \\
\hline \multirow{3}{*}{ dB2 } & Sensitivity & 91.6583 & 92.3204 & 90.7522 & 91.0144 \\
\cline { 2 - 6 } & Specificity & 94.9965 & 93.9573 & 93.5633 & 93.6701 \\
\cline { 2 - 6 } & Average Detection & 93.3275 & 93.1388 & 92.1578 & 92.3423 \\
\cline { 2 - 6 } & MSE & 0.00575 & 0.00400 & 0.00483 & 0.00368 \\
\hline \multirow{3}{*}{ db4 } & Sensitivity & 92.2134 & 88.1806 & 92.2523 & 90.6058 \\
\cline { 2 - 6 } & Specificity & 95.731 & 93.9106 & 94.9850 & 95.5017 \\
\cline { 2 - 6 } & Average Detection & 93.9722 & 91.0457 & 93.6185 & 93.0537 \\
\cline { 2 - 6 } & MSE & 0.00519 & 0.00608 & 0.00377 & 0.00583 \\
\hline & Sensitivity & 93.3694 & 92.3381 & 89.7018 & 92.0051 \\
\cline { 2 - 6 } & Specificity & 95.9438 & 94.2282 & 93.4793 & 91.5050 \\
\cline { 2 - 6 } & Average Detection & 94.6565 & 93.2831 & 91.5905 & 91.7550 \\
\cline { 2 - 6 } & MSE & 0.00712 & 0.00451 & 0.00477 & 0.00544 \\
\hline
\end{tabular}

in Heursure Soft Thresholding method outperformed other three methods in terms of average detection and MSE values. For a given low value of MSE all four wavelets against four Soft Thresholding methods provided more than $92 \%$ of average detection. This high level performance is achieved due to the effect of threshold in the extracted feature to remove the noisy components.

It is observed from the Table IV. that the proposed method has higher accuracy than the other standard methods. It was found that dB4 gave slightly better result with compared to db2. This corroborated the finding by [1] that db4 is the most suitable wavelet to be use in the task of EEG signals analysis. As stated in [1], the wavelets of lower order are too coarse to represent the EEG signals that have many spikes, while higher order wavelets oscillate too wildly and this characteristic is not desirable as the

Table IV. Analysis of ANN based Feature Selection Methods and the Achieved Performance.

\begin{tabular}{|c|c|c|c|c|c|}
\hline Reference & Method & $\begin{array}{l}\text { Objective of the } \\
\text { Study }\end{array}$ & $\begin{array}{l}\text { EEG data } \\
\text { type }\end{array}$ & $\begin{array}{l}\text { Number } \\
\text { of classes }\end{array}$ & $\begin{array}{l}\text { Classification } \\
\text { accuracy }\end{array}$ \\
\hline $\begin{array}{l}\text { T Zallas } \\
2007\end{array}$ & ANN & $\begin{array}{l}\text { Time Frequency } \\
\text { analysis }\end{array}$ & $\begin{array}{l}\text { Continuous } \\
\text { EEG }\end{array}$ & 4 classes & 92.4 \\
\hline $\begin{array}{l}\text { Guo L } \\
\text { etal } 2010\end{array}$ & MLP & $\begin{array}{l}\text { DWT with line } \\
\text { length feature }\end{array}$ & $\begin{array}{l}\text { Discrete } \\
\text { EEG }\end{array}$ & 6 classes & 91.5 \\
\hline $\begin{array}{l}\text { Orhan U } \\
\text { Etal } 2011\end{array}$ & MLP & $\begin{array}{l}\text { DWT with K- } \\
\text { means algorithm }\end{array}$ & $\begin{array}{l}\text { Discrete } \\
\text { EEG }\end{array}$ & 4 classes & 94.8 \\
\hline $\begin{array}{l}\text { Proposed } \\
\text { method }\end{array}$ & WNN & $\begin{array}{l}\text { DWT with multi } \\
\text { level Thresholing }\end{array}$ & $\begin{array}{l}\text { Continuous } \\
\text { EEG }\end{array}$ & 9 classes & 96.33 \\
\hline
\end{tabular}


wavelets cannot represent the EEG signals well.

Table V. Shows that the FPGA implementation of WNN is consumed $60 \%$ of total time when compared to Mat Lab version of WNN. Therefore this FPGA model is quick in classification and response in the Epilepsy detection.

Table V. Comparison of computation Time of WNN in Mat Lab and FPGA.

\begin{tabular}{cll}
\hline Total Patients & Model & Total Time in sec \\
\hline \multirow{2}{*}{ 20 patients } & WNN without PSO Learning in Mat Lab7 & 75.128 \\
\cline { 2 - 3 } & $\begin{array}{l}\text { WNN with PSO Learning in FPGA(vertex 3 } \\
\text { board) }\end{array}$ & 42.38 \\
\hline
\end{tabular}

\section{Conclusion}

In this paper, implementation of Wavelet Neural Networks with PSO learning ability using FPGA was proposed. Some of the features of the Wavelet Neural Networks with the PSO algorithm can be summarized as follows: (1) an analog WNN is realized based on digital circuits; (2) hardware implementation of the PSO learning rule is relatively easy; and (3) hardware implementation can take advantage of parallelism. The WNNs models with varied activation functions and different feature extraction techniques were investigated in the task of severity index of epileptic seizure classification. Based on the overall classification accuracy obtained, the Haar wavelet was found to be the best wavelet function to be used. The db4 was also found to be more suitable to be used compared to db2. By replacing the extreme values of wavelet coefficients with suitable percentiles, the classifiers gave better classification accuracy. A major drawback of the existing WNN is that their application domain is limited to static problems due to their inherent feedforward network structure. The future research will be in the direction to solve this static structure problem through recurrent WNN and Extreme Learning Machine. 